\title{
PENSANDO LA NATURALEZA DE LA MONEDA DESDE UNA PERSPECTIVA CRÍTICA POSFUNDACIONAL
}

Camilo Andrés Guevara Castañeda Universidad Nacional de Colombia

Colombia 
Camilo Andrés Guevara Castañeda

JEL: E40, E50

\title{
Pensando la naturaleza de la moneda desde una perspectiva crítica posfundacional
}

\section{Resumen}

El artículo busca rescatar y reformular una forma de pensar problemas epistemológicos en economía, específicamente en torno al debate de la naturaleza del dinero. Se presentan las dos concepciones antagónicas sobre la naturaleza del dinero; la del dinero-mercancía y la del dinero-deuda. Después se presenta la propuesta ontológica de Tony Lawson que articula las dos concepciones: el dinero no es en sí mismo mercancía o deuda, sino que estas formas son posicionamientos sociales del dinero. Se recogen algunas críticas a esta propuesta. Por último, dentro del espíritu de corrientes como la fenomenología y el pragmatismo, se propone un antiesencialismo crítico. Se argumenta a favor del dinero como relación social, por los mundos que se abren y las consecuencias que se producen.

Palabras clave: Naturaleza de la moneda, Dinero mercancía, Dinero deuda, Posicionamiento social

\section{Réflexion sur la nature de la monnaie dans une perspective critique post-fondation}

\section{Résumé}

L'article cherche à sauver et à reformuler une façon de penser les problèmes épistémologiques en économie, notamment autour du débat sur la nature de l'argent. Les deux conceptions antagonistes de la nature de l'argent sont présentées; celle de l'argent-marchandise et celle de l'argent-dette. On présente ensuite la proposition ontologique de Tony Lawson qui articule les deux conceptions: l'argent n'est pas lui-même une marchandise ou une dette, mais ces formes sont des positions sociales de l'argent. Certaines critiques de cette proposition sont collectées. Enfin, dans l'esprit de tendances telles que la phénoménologie et le pragmatisme, un anti-essentialisme critique est proposé. Elle est argumentée en faveur de l'argent en tant que relation sociale, pour les mondes qui s'ouvrent et les conséquences qui se produisent.

Mots clés: Nature de la monnaie, argent des marchandises, argent de la dette, positionnement social

\section{Thinking about the nature of currency from a post-foundation critical perspective}

\begin{abstract}
The article seeks to rescue and reformulate a way of thinking about epistemological problems in economics, specifically around the debate on the nature of money. The two antagonistic conceptions about the nature of money are presented; that of money-merchandise and that of money-debt. Then Tony Lawson's ontological proposal is presented that articulates the two conceptions: money is not itself merchandise or debt, but these forms are social positions of money. Some criticisms of this proposal are collected. Finally, within the spirit of trends such as phenomenology and pragmatism, a critical anti-essentialism is proposed. It is argued in favor of money as a social relationship, for the worlds that open and the consequences that occur.
\end{abstract}

Keywords: Nature of currency, merchandise money, debt money, social positioning 


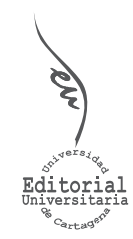

Artículo de Investigación

\section{Pensando la naturaleza de la moneda desde una perspectiva crítica posfundacional'.}

\section{INFORMACIÓN DEL ARTÍCULO}

Recepción del artículo: 10/10/2018

Concepto de evaluación: 15/11/2018

Aceptación del artículo: 17/12/2018
Camilo Andrés Guevara Castañeda*

Universidad Nacional de Colombia, Colombia

\section{INTRODUCCIÓN}

En las últimas dos décadas hemos presenciado un nuevo impulso en el número de publicaciones (artículos y libros) que nos invitan a repensar la naturaleza del dinero (Ingham, 2004; Graeber, 2011; Martin, 2013; Dodd, 2014; Aglietta, Ponsot y Ould Ahmed, 2016). Por supuesto, este interés no es nuevo, pero la crisis de 2008 , el surgimiento de las criptomonedas y las monedas locales y complementarias, han dado un nuevo impulso a la necesidad de volver a pensar las preguntas fundamentales. Así, cuestiones cómo ¿Qué es el dinero? ¿Cómo surge? ¿Para qué sirve? ¿Por qué existe? y ¿Cómo se establece su valor? han vuelto a tener una mayor discusión y relevancia. En el debate sobre la naturaleza del dinero se suelen oponer dos grandes concepciones, aunque al interior de cada concepción hay matices y diferenciaciones. Grosso modo se opone la concepción del dinero como una mercancía (cosa) y la del dinero como una relación social de deuda. Estas dos concepciones, a su vez están relacionadas con que función del dinero es predominante, si la función de medio de cambio o la de unidad de cuenta, y que tan importante es el poder del mercado o el Estado en el origen y evolución de la moneda.

Este es un debate en el que han intervenido antropólogos, sociólogos, filósofos y economistas entre otros, lo que ha tornado el debate sobre la naturaleza del dinero en una conversación interdisciplinar. No obstante, como señala acertadamente John Smithin (2013), el estudio del dinero debe trascender esta interdisciplinariedad. Smithin utiliza

\footnotetext{
*Autor de correspondencia: caguevarac@unal.edu.co

1. Agradezco las conversaciones y comentarios de los profesores Bruno de Conti y David Dequech del Instituto de Economía de la Universidad Estatal de Campinas (Unicamp). También comentarios de Luis Álvaro Gallardo y Álvaro Moreno. Por supuesto ellos no son responsables de lo aquí propuesto.
} 
la expresión ciencia monetaria, para tratar de evitar el reduccionismo cuando pensamos el dinero como hecho social complejo y nos invita a pensar el dinero desde cuatro metaniveles; una dimensión ontológica, una epistemológica, una ética y una política. Siguiendo estos cánones, identifica la dimensión ontológica con el programa de investigación de la ontología social realista (Searle, 2010) (Lawson, 1997); la dimensión epistemológica tanto con el análisis de la sociología económica como con la macroeconomía monetaria y por último, la dimensión ética y política son abarcadas por la economía política (Smithin, 2013).

Este artículo acepta el desafío de pensar el dinero más allá del reduccionismo, pero busca cuestionar y problematizar el entendimiento de lo que Smithin llama "dimensión ontológica". Al contrario de su propuesta, se propone una interpretación antiesencialista y pragmática, que discute el proyecto de la ontología social, y que se justificará cómo más pertinente para dar cuenta de lo que aquí proponemos llamar la "política del dinero". Es decir, los conflictos que surgen por tratar de constituir la moneda con determinada potencialidad (la del dinero mercancía o el dinero deuda). Esta política del dinero se ubica en lo que Oliver Marchant ha llamado el posfundacionalismo político².

La discusión empieza entonces con Nigel Dodd, quien en su estudio The Social Life Of Money (2014), no ve razón alguna para optar por una u otra conceptualización sobre la naturaleza del dinero. Dodd nos invita a pensar el dinero como un espacio abierto, sabiendo de antemano que ninguna conceptualización tiene la llave para resolver finalmente el misterio de la naturaleza de dinero. Otros como Zelmanovitz (2016) rechazan la tesis de Dodd y afirman que el antiesencialismo equivale a no aportar nada nuevo al debate y es una posición débil e insostenible.

En este artículo me interesa debatir la posición de Zelmanovitz y reformular la de Dodd, pues como veremos, el antiesencialismo es de vital importancia en un mundo que ha naturalizado los cimientos del orden vigente o que apela a la supuesta naturaleza de las cosas para imponerse; es vital para un mundo que se niega a discutir los fundamentos de su construcción y que no puede ver la contingencia de los mismos. El esencialismo y el naturalismo se ven expresados cotidianamente con los slogans, ¡No hay alternativa!, ¡No hay otra manera!, ¡No se puede!, frases que por supuesto abundan cuando de política monetaria se trata. Por lo tanto, el antiesencialismo abre la posibilidad de deconstruir cualquier tipo de fundamentación. No obstante, a diferencia de la argumentación de Dodd, es necesario un posfundacionalismo al activar otros mundos posibles que deben ser defendidos no desde su supuesta correspondencia con la naturaleza de las cosas, sino por los mundos que se pueden traer a la mano; en otras palabras y en clave pragmatista, por las consecuencias que produce determinada concepción. Por supuesto, esto abre otro tipo de preguntas. Si hablamos de consecuencias ¿de qué tipo?, ¿para quienes?, ¿para fomentar que tipo de sociedad? Es en la respuesta a estas preguntas donde se puede reconstruir un horizonte crítico de un pensamiento posfundacionalista. No se trata entonces de juzgar a-priori una concepción sobre la naturaleza del dinero, aunque dicho camino pueda emplearse y pueda ser valioso, como de mostrar a-posteriori que las concepciones tienen diferentes alcances y que no generan potencialmente la misma realidad.

El articulo está entonces estructurado de la siguiente manera. En la segunda sección revisamos la concepción del dinero mercancía y en la tercera la del dinero como relación 
social. En la cuarta la manera como se articula el dinero desde la ontología social realista y en la quinta se desarrollan algunas críticas a la ontología social. Por último, en la sexta sección se esboza la idea de pensar el dinero desde una perspectiva antiesencialista. El articulo cierra con algunas conclusiones.

\section{EL DINERO-MERCANCÍA}

Es importante comenzar señalando que hay varias versiones de lo que se ha venido a llamar la teoría del dinero mercancía. Así, es preciso tener presente que cuando nos referimos a la teoría del dinero mercancía, hablamos de un tipo ideal (Weber, 2006) del que es posible extraer algunos rasgos fundamentales a las que sin duda adhieren la mayoría de las vertientes.

Comencemos con el registro histórico. Respecto al origen del dinero suele contarse la historia de la evolución del trueque hasta llegar a la moneda. Una de estas historias, es brindada en un texto reciente por el profesor de Columbia Xavier Sala-i-Martín (2015), quien nos dice que el dinero aparece alrededor del año 3000 a.C en Sumeria en forma de cereal (cebada) y que la gente aceptaba el pago de su salario en esta forma, no porque se fueran a comer la cebada, sino porque era un medio de cambio para poder comprar todos los productos que querían y podían consumir. Sin embargo, señala Sala-i-Martin, la cebada tenía dos problemas; que era difícil transportarla y que se iba deteriorando con el paso del tiempo. La cebada fue abandonando la escena (o el objeto dinero fue evolucionando) y se pasó al siclo de plata (Polvo), que era más liviano que el anterior, pero precisaba de una balanza en cada momento para pesar el polvo y así poder hacer equivalente las transacciones. No fue sino hasta el año 640 a.C que se inventaron las monedas y bajo el dominio del imperio romano que se expandieron.

Este tipo de narrativa ha sido uno de los ejes fundamentales para comprender el dinero. Como señala Geoffrey Ingham (2004), la teoría del dinero-mercancía es bastante antigua y pueden encontrarse algunos registros en las descripciones de Aristóteles sobre la evolución y origen del dinero. En esta conceptualización inicial, se suele identificar el dinero como algo material y tangible, como por ejemplo el oro y la plata que son esenciales para constituirlo (Ingham, 2004). Partiendo de algunos registros históricos, analíticos y teóricos, que pueden variar según la vertiente de la teoría, los partidarios del dinero mercancía nos señalan que lo que subyace a una economía que utiliza dinero, no es otra cosa que una economía de trueque. En el fondo, estamos ante un mecanismo en el cual son las mercancías, o mejor, una de las mercancías la que se vuelve patrón, y que compra otras mercancías. Así, para Jevons (1875), el dinero es precisamente un medio que permite superar los problemas asociados a la doble coincidencia de necesidades que plantea el trueque. Desde esta perspectiva, si yo deseo algo que él otro tiene para cambiar, pero este no desea lo que yo tengo, nos vemos enfrentados a un problema de coincidencia de necesidades y el intercambio no tiene lugar. La mercancía dinero pretende así superar este obstáculo.

Por supuesto no todos están de acuerdo en la manera en la que se cuenta esta historia. Algunos economistas austriacos, por ejemplo, están en desacuerdo en que se los ubique compartiendo el origen del dinero con el trueque. Para ellos, los deseos de los individuos de conseguir bienes y servicios son el motor que hace aparecer el dinero; es decir, un resultado no buscado, un orden espontaneo, que emerge de las interacciones de los individuos que terminan adoptando por ensayo y error, la mercancía más vendible como medio de cambio (Menger, 1892). Este resultado no es fruto de un diseño intencionado, como lo podría ser una ley estatal que declara la unidad de cuenta (pesos, dólares, etc.) de 
un país, si no que es un proceso evolutivo y emergente en el que el estado solo vendría a legitimar un proceso pre-existente. Se afirma así, la primacía de la función de la moneda como medio de cambio, que no es un producto de la soberanía estatal. Los economistas austriacos han refinado la historia básica de Menger, interpretándola con el concepto de costo de transacción, donde el costo fundamental es el de la información. El dinero es un resultado no buscado que logra reducir los costos de transacción, para facilitar el trueque de los productores y así poder ampliar la división del trabajo (Zelmanovitz, 2016). Otra variante ha desarrollado los modelos de búsqueda, que tratan de mostrar como el dinero emerge de las interacciones individuales, en las que se van descartando al final de cada periodo, las mercancías que son candidatas a dinero.

Por su parte, la versión neoclásica ${ }^{3}$ conceptualizada en profundidad por Patinkin, muestra que el valor del dinero tiene que explicarse por el principio de la utilidad marginal, como cualquier otra mercancía. Esto supone que el dinero como medio de intercambio tiene valor en sí mismo. Sin embargo, esto ha generado problemas internos de coherencia a la versión neoclásica, pues si el dinero tiene utilidad, debería aumentar también la riqueza real de los que intercambian, lo cual no ha podido probarse. La razón de fondo como ha sugerido Frank Hahn, es que el dinero se ha introducido a-posteriori en un sistema, consistente con la maximización de la utilidad y que ya se encuentra en equilibrio general (Hahn, 1965). Es como si tuviéramos un rompecabezas armado completamente y nos quedara faltando una pieza. ¿Cómo puede ubicarse entonces? Además, si pudiera encontrarse un lugar para que el dinero no distorsionara el equilibrio general, existiría el problema de su significado, pues el dinero entraría en este esquema como una forma en la que se tiene riqueza y no para ser intercambiado por bienes. Se tiene entonces la inconsistencia de que la utilidad del dinero surge de su rol como forma de tener riqueza, mientras que el valor del dinero surge de su rol como medio de intercambio.

Otro elemento importante de la versión neoclásica, es la exogenidad de la oferta de dinero (Parguez \& Seccareccia, 1999). Este principio es clave porque la exogenidad de la oferta monetaria está relacionada con la escasez de dinero, y la escasez debe existir para que el dinero tenga valor. A su vez, si la oferta y la demanda de dinero no son independientes, no existe un solo punto en el cual se logre un equilibrio para una curva de oferta dada (Keen, 2011), a menos que se rescate el equilibrio con nociones como la tasa natural de interés, como lo hacen los modelos del nuevo consenso macroeconómico (New Keynesians \& New Classicals) que adoptan la endogeneidad del dinero sin un teoría endógena del dinero (Lavoie, 2014).

La concepción del trueque en la que el dinero es algo posterior al orden mercantil, es una de las imágenes más potentes de la economía neoclásica. Está presente en la ley de Say, la ley de Walras, la teoría cuantitativa del dinero, la neutralidad del dinero, la teoría de los fondos prestables etc.

\footnotetext{
3. Con el surgimiento y profundización de diferentes subcampos como la economía del comportamiento, el neoinstitucionalismo, la nueva economía de la información, la economía evolutiva, la economía experimental, etc. suele argumentarse que la economía neoclásica ha muerto (Colander, 2000). Sin embargo, cuando aquí se habla de teoría neoclásica, se refiere a los principios metodológicos en la construcción de conocimiento como el Individualismo metodológico, el instrumentalismo y la hiperacionalidad o su modelo de desviación de racionalidad ver (Gigerenzer, 2010). Un neoclásico típico acepta entonces elementos como 1) la teoría de la productividad marginal. 2) La tasa natural de desempleo 3) Tasa natural de interés 4) la teoría de los fondos prestables 5) Efectos Crowding-Out 6) la hipótesis de mercados eficientes, 7) Reservas bancarias como causas de los depósitos y los créditos bancarios 8) Exceso de oferta de dinero como causa de la inflación 9) La ley de Say 10) Empleo agregado se determina en el mercado de trabajo 11) Salarios pegajosos como la causa del desempleo 12) Costos unitarios en forma de U (Lavoie, 2014).
} 
Así, si el dinero es exógeno "nunca puede ser impulsado por el crédito... se supone que los bancos no crean dinero porque se los considera como intermediarios financieros puros, con el suministro de dinero determinado por las acciones exógenas del banco central, como en el modelo monetarista estándar (Parguez \& Seccareccia, 1999; Missaglia, 2015; Escobar \& Guevara, 2017). Los bancos no crean dinero, y el crédito es esencialmente una transferencia de un poder de compra ya existente en la economía, lo que nos lleva a la idea de que el crédito no puede desestabilizar el mecanismo de mercado, pues esta es intrínsicamente estable. La inestabilidad solo puede ser exógena, como por ejemplo en los modelosRBC donde el cambio tecnológico y los cambios en la productividad sacan a la economía del equilibrio. Así, lo fundamental para los economistas neoclásicos es que en el largo plazo el mercado se vacía o está en equilibrio, como sucede en el trueque. Esto implica que el dinero también es neutral en el largo plazo, porque si el dinero actúa como en el trueque, no debe afectar las variables reales, es decir, los precios relativos o razones de intercambio cuando hay equilibrio.

Esta visión que se construye sobre la imagen del trueque somete e intenciona la realidad hacia el horizonte trazado por esta imagen. En su versión contemporánea, las expectativas son el vehículo para producir los equilibrios, de allí que hoy sean tan importantes las expectativas que están guiadas entre otros por los bancos centrales al anunciar las metas y comprometerse en cumplirlas. Una verdadera profecía autocumplida.

\section{EL DINERO COMO RELACIÓN SOCIAL}

En este apartado bajo lo que se denomina el dinero como relación social, se agrupan varias variantes que podrían aparecer como contradictorias pero que tienen más elementos en común que divergencias (Dequech, 2013). Se trata de la teoría del dinero crédito, las dos variantes de la teoría del dinero estatal, y la teoría convencionalista de la moneda (Aglietta \& Orleán, 1988).

¿Qué tienen en común estas teorías? Cómo sugiere Paul Davidson:

"La mayoría de las disputas entre varias escuelas de pensamiento sobre el papel del dinero en un sistema económico se deben a las diferentes conceptualizaciones de las funciones y propiedades del dinero y sus relaciones con el paso del tiempo. El tiempo es un dispositivo que evita que todo suceda a la vez" (Davidson, 2007, pág. 139)

Las teorías señaladas anteriormente comparten una concepción temporal en la que el tiempo es un fenómeno irreversible. Existe una diferencia entre pasado y futuro, lo que significa que el tiempo tiene una flecha temporal. Las consecuencias de este reconocimiento son enormes pues se reconoce a plenitud la incertidumbre ${ }^{4}$, pero también la concatenación de pasado y presente (Tiempo histórico). Como resume Giuseppe Fontana:

"Contra la opinión de que las economías capitalistas son una especie de economía multi-trueque en la que todas las transacciones ocurren simultáneamente (tiempo lógico), que la oferta monetaria cambia por capricho del banco central

\footnotetext{
4. Paul Davidson, vía Keynes, muestra por ejemplo que la demanda de dinero tiene sentido por la incertidumbre (Davidson, 1972). Pero también es clave para explicar la no soberanía de los deseos y las creencias de los seres humanos en la teoría mimética de la moneda.
} 
(dinero exógeno) y solo afecta, vía la teoría clásica cuantitativa, las variables en el sector monetario (el dinero es neutral), teóricos del dinero endógeno han argumentado que el dinero y la producción son características esenciales de las economías modernas. La producción es un proceso dinámico y el dinero se filtra necesariamente en la historia de un movimiento hacia la creación y distribución de bienes y servicios. El dinero ingresa al sistema económico a través de las iniciativas privadas de sus principales agentes y modifica el funcionamiento interno de la economía (del sector real)" (Fontana, 2004, pág. 10)

En segundo lugar, bajo esta concepción de la moneda como relación social se rechaza con fuerza la idea de que el orden mercantil es primero y la moneda se introduce después. Se cuestiona la idea de que "en el principio fueron los mercados" (Williamson, 1975, pág. 20) y se parte reconociendo la naturaleza social del origen del dinero (Ingham, 2004). Por supuesto, la historia puede variar dependiendo el énfasis o las dimensiones que se aborden.

En algunas vertientes, como, por ejemplo, la del dinero-crédito o deuda, se cuestiona antropológicamente la historia tradicional del trueque (Graeber, 2011). David Graeber en su famosa obra En deuda (2011), cuestiona la idea de Adam Smith sobre la evolución del dinero, contada en la sección anterior en la versión de Sala-i-Martin (2016). Para Graeber, desde el principio en el intercambio lo que ha existido son relaciones de deuda que se han registrado mediante jeroglíficos o tablas y que puede evidenciarse en el registro arqueológico en Mesopotamia, donde se sabe que existían mecanismos de perdón o jubileo de las deudas (Graeber, 2011).

Desde la perspectiva del dinero crédito, que se conceptualiza tempranamente en autores como Alfred Mitchel Innes (1914), el dinero aparece como una promesa de pago. "Una moneda de oro es solamente una promesa de pagar algo equivalente a una moneda de oro" (Graeber, 2011, pág. 40) o como dice Georg Simmel en su gran obra La filosofía del dinero, "el dinero es el valor de las cosas, sin las cosas mismas...la relación entre cosas que persiste a pesar del cambio entre las cosas mismas" (Simmel, 2014, págs. 121-124). En otros términos, es un valor abstracto, un patrón de medida que requiere de creencias como las de un feligrés a su religión (Simmel, 2014). Esto porque si bien la unidad de cuenta (dinero) puede establecerse teniendo como referente algún tipo de medida ya establecida, como el peso de algunas cosas materiales (polvo), como en las historias del dinero mercancía, "la existencia de un patrón invariante de valor es una medida abstracta, es decir, "existe en el pensamiento en lugar de la materia" (Ingham, 2017, pág. 7)

Otro elemento común a las teorías del dinero como relación social es la predominancia y existencia primaria que tiene la función unidad de cuenta sobre la función de medio de cambio. Como dice Ingham: "Es un principio constitutivo de la teoría del crédito que la capacidad de las 'cosas' para portar o transmitir el valor monetario, está constituido enteramente por su denominación en una unidad de cuenta" (Ingham, 2017, pág. 3). En este punto, aparece una conexión fuerte con las teorías del dinero estatal. Desde la perspectiva del dinero estatal, que se conceptualiza en autores como Georg Knapp (1905) y anteriormente en autores de la escuela histórica alemana, el dinero aparece como una creación del Estado. La moneda aparece como un vínculo social que es posible por una soberanía y una legitimidad que al denominar la unidad de cuenta posibilita la sociedad.

Dentro de la teoría del dinero estatal tenemos dos variantes. La primera ligada a los contratos y la segunda relacionada con los impuestos. En la primera se argumenta que "el dinero es tanto la unidad de cuenta en la que se denominan los contratos, como los 
medios para el descargo de las obligaciones contractuales" (Dequech, 2013, pág. 263). Esta visión, liderada por Davidson, argumenta que el dinero y los contratos monetarios existen al mismo tiempo. Así, toda transacción con dinero es explícita o implícitamente un contrato. Se señala además que todas las relaciones fundamentales capitalistas están mediadas por contratos, como por ejemplo los contratos entre empleados y trabajadores (Dequech, 2013). Así, los contratos y el dinero se comportan como un entrelazamiento cuántico, un fenómeno en el cual aunque dos partículas se encuentren separadas en el espacio, deben describirse mediante un estado único que involucra a las dos partículas. En la segunda variante de la teoría del dinero estatal se argumenta que "el dinero es tanto la unidad de cuenta en la que se denominan los impuestos, y que es aceptado como medio para el descargo de las obligaciones tributarias" (Dequech, 2013, pág. 266). Esta visión, argumenta que el dinero es conducido por los impuestos. Así, son clave los vínculos "entre soberanía política y autoridad fiscal de un lado y la creación monetaria, acuñación y el Banco central por el otro" (Goodhart, 1998, pág. 3). Desde esta concepción, cambia el sentido, el significado y la causalidad existente entre impuestos y gasto público y allí reside una de sus consecuencias importantes.

Por último, tenemos la teoría convencionalista del dinero. Uno de sus proponentes, André Orleán, recurre a la teoría de la mimesis para mostrar como las preferencias y la mercancía elegida como dinero por cada individuo no se anclan en la soberanía de la elección de una conciencia individual, sino que depende de las preferencias y la mercancía elegida por los otros. En este modelo los otros aparecen como guías, pero también como rivales. El otro es modelo a seguir, porque no hay soberanía en mis deseos, pero cuando el otro desea lo que yo deseo también se engendra una tensión de rivalidad (Aglietta \& Orleán, 1988).

Orleán muestra que es este proceso convencionalista (el dinero puede ser cualquier mercancía) y mimético el que genera cierta estabilidad en la mercancía elegida como dinero. Por el contrario, el modelo del deseo soberano en el que cada uno tiene una mercancía candidata a dinero, no puede generar una mercancía estable como dinero, sino que genera un caos. Es entonces la imitación la que produce la autotrascendencia (Dupuy, 2012) del proceso mimético y aparece un orden estable que se cree exógeno. Este orden se revela aún más estable con la legitimidad del estado, al establecer la unidad de cuenta, pero que no sirve de nada si toda la sociedad pierde la confianza en el dinero decretado legalmente. La teoría de Orlean, aunque parece iniciar con los deseos de los individuos como la versión austriaca, tiene una divergencia fundamental y es que el proceso mimético introduce las relaciones sociales en el corazón de los individuos, pues el individuo no se afirma autónomamente.

Por otro lado, aunque pareciera que las teorías del dinero estatal contradicen el convencionalismo, se puede mostrar que hay más continuidad que ruptura. David Dequech muestra, que tanto la variante de los contratos como la de los impuestos, no tendrían problema en aceptar que aquello que sirve como medio de descargo de obligaciones es una convención (Dequech, 2013). Es más pertinente visualizar estas teorías como parte de una matriz y no como teorías aisladas, sin que esto implique negar las diferencias, ni los debates entre "teorías cercanas".

Antes de terminar, quisiéramos resumir y profundizar algunas consecuencias que se derivan de la conceptualización de la naturaleza del dinero como relación social. Por supuesto en detalle las consecuencias dependen de cada variante. No obstante, en términos generales pensar la naturaleza del dinero bajo las coordenadas de relaciones 
sociales, cambia las perspectivas de lo que es posible o no hacer en el mundo. Cambia la forma de valoración sobre el para qué de la moneda y el tipo de arreglos institucionales considerados valiosos. Como dice recientemente Ann Pettifor (2017) "La teoría monetaria de Keynes y las políticas asociadas serán vitales para la restauración de la estabilidad económica y ambiental y para la restauración de la justicia social" (Pettifor, 2017, pág. 17) Uno de los elementos centrales es que, bajo esta concepción, la noción de que el dinero es escaso en sí mismo se rechaza. Como dice Keynes: "nos podemos permitir, todo aquello que seamos capaces de hacer" (citado en Pettifor, 2017, pág. 25). Así, desde esta perspectiva las restricciones no son presupuestales. Los bancos comerciales no se ven limitados por la cantidad de reservas o depósitos que tienen para hacer préstamos. El sistema crédito implica que se crea un poder de compra nuevo y no uno que se traslada. Es como un mago que por medio de un truco trae recursos del futuro. Este sistema se creó contra la usura, en el cual los prestamos si se hacían con respaldo en los ahorros y excedentes de algunas personas, lo cual limitaba bastante la expansión de proyectos productivos en la sociedad. Hoy por supuesto, la producción social del dinero ha sido capturada por los banqueros quienes han configurado la peor de las perspectivas para la sociedad, la del dinero fácil y caro (Pettifor, 2017). Se otorgan préstamos en proyectos con pocas perspectivas de repago y con altas tasas de interés, lo que genera un peligroso coctel, además que la sociedad se ve restringida en los proyectos que puede conseguir.

A su vez, la Teoría Monetaria Moderna (MMT) que aquí se presentó como la vertiente tax-driven approach de la teoría del dinero como criatura del estado, nos dice que el gasto del gobierno antecede a los impuestos, por lo que los impuestos no financian el gasto. Se invierte así la causalidad y se rechaza la lógica con la cual se justifican muchas reformas tributarias: esto es, que el gobierno recaude fondos para financiarse. Por supuesto, la implementación de una concepción de este estilo puede verse restringida desde su lado operativo de varias maneras, incluyendo las creencias de los agentes, o en países con monedas periféricas, monedas que internacionalmente no cumplen sus funciones, se enfrentan restricciones que dependen de la posición del país en el Sistema Monetario Internacional (Vergnhanini \& De Conti, 2017). No obstante, la naturaleza del dinero adoptada cambia lo que puede hacerse (con restricciones) o no, lo que puede verse y analizarse y lo que no. Por ejemplo, la no neutralidad del dinero en el corto y largo plazo, derivada de esta concepción, implica que el dinero tiene consecuencias en las variables reales de la economía. El vínculo entre dinero, producción, empleo y distribución es así fundamental.

Como hemos visto a lo largo de esta sección, las variantes anteriormente vistas comparten un rechazo profundo a la naturaleza ofrecida por el dinero-mercancía. Como resumió lucidamente Geoffrey Ingham:

"La naturaleza del dinero como poder adquisitivo y de endeudamiento es una fuerza social abstracta que solo es aparente en su operación. Su poder no reside en su estructura material; estos solo son los diferentes medios por los cuales estos poderes se consideran establecidos como valor estándar, ya sea como deposito de valor o solamente transmisor. El dinero está en la categoría de poderes construidos socialmente que están representados por objetos y performados, es decir, hecho 'real' por las relaciones sociales y las instituciones" (Ingham, 2017)

\section{LA NATURALEZA DEL DINERO BAJO LA ÓPTICA DEL POSICIONAMIENTO SOCIAL (ONTOLOGÍA SOCIAL)}

En años recientes, el reconocido filosofo de la ciencia económica Tony Lawson ha lanzado el desafió de repensar la naturaleza del dinero bajo la óptica de la ontología social 
por medio del posicionamiento social. Para entender su perspectiva hacemos una breve introducción a esta concepción y sus raíces.

Comencemos por señalar que los caminos que recorren los denominados proyectos ontológicos (ontología social, status ontológico, etc.) derivan de la concepción de la ciencia que ha trazado el Realismo Crítico (RC). Como lo señala Roy Bhaskar uno de los fundadores de esta concepción:

Las cosas existen y actúan independientemente de nuestras descripciones, pero solo podemos conocerlas bajo descripciones particulares. Las descripciones pertenecen al mundo de la sociedad y de los hombres; los objetos pertenecen al mundo de la naturaleza. Expresamos [nuestra comprensión de] la naturaleza en el pensamiento. La ciencia, entonces, es el intento sistemático de expresar en el pensamiento las estructuras y formas de actuar de las cosas que existen y actúan independientemente del pensamiento. El mundo es estructurado y complejo y no está hecho para los hombres. Es completamente accidental que existamos, y entendamos algo sobre nuestra vida en él. (Baskhar, 1975, pág. 250)

El realismo crítico es entonces una nueva apuesta para dar con la naturaleza última de la realidad. Se trata de encontrar cuales son las estructuras fundamentales que tienen propiedades y poderes causales (se dirá que son causalmente eficaces) y que constituyen y "producen" realidad.

Los proyectos ontológicos son una de las expresiones de esta concepción realista de la ciencia. Se dice por ejemplo que "La ontología social examina la naturaleza y la estructura básica de la vida social y los fenómenos sociales” (Schatzki’s, 2005, pág. 465).

Una de las herramientas desarrolladas en el marco de la ontología social es el posicionamiento social. En palabras de Tony Lawson, uno de los defensores de la centralidad de esta herramienta para la teoría social:

Mi punto de partida es una evaluación, a menudo defendida, de que la realidad social está constituida en gran parte por las totalidades emergentes, donde las personas y las cosas (ampliamente concebidas) se incorporan como componentes...El posicionamiento social es el término para el proceso mediante el cual, a través de la aceptación general a través de una comunidad, individuos humanos, cosas u otros fenómenos se incorporan como componentes de estas totalidades emergentes. En todos los casos, posición social implica la aceptación generalizada de los siguientes tres elementos con respecto a cualquier artículo que se posicione: (i) la asignación de un estado acordado; (ii) su ubicación práctica como un componente de una totalidad; y (iii) el aprovechamiento de algunas de sus capacidades ya poseídas para servir como una o más funciones del sistema de la totalidad" (Lawson, 2016, pág. 964).

Lawson pone el ejemplo en el que los seres humanos pueden ser posicionados, en el espacio de una universidad, como profesores, asistentes o estudiantes entre otros. En el caso de un profesor, tenemos por ejemplo una identidad acordada (cuando alguien gana un concurso para ser profesor), una ubicación practica (el profesor hace parte de la red de profesores e investigadores de la universidad), en la que también se adquieren derechos y obligaciones al ser emplazado en una posición. Ser profesor da derecho a exigir tareas y trabajos, mientras que ser estudiante a la obligación de hacerlos. Por último, la capacidad oral de un profesor sirve para dar una buena clase y cumplir con el objetivo de enseñar. Lawson señala que, en la mayoría de los ejemplos del posicionamiento social, el ocupante y la posición pueden confundirse, pero que es necesario distinguirlos. En este caso 
profesor es tanto la posición en una universidad, como lo que es la persona (ser profesor). El enfoque del posicionamiento social apuesta entonces a señalar unas estructuras en las que se emplazan los individuos, generando así unas inter-relaciones entre agencia (las personas que tienen unas capacidades) y estructura (la posición profesor que se ocupa en la universidad)

Así, Lawson propone repensar la naturaleza del dinero bajo la óptica del posicionamiento social y con el mismo objetivo que se ha trazado desde hace tiempo (1998): demostrar que las diferentes escuelas heterodoxas comparten la misma ontología. Como se sabe, la forma de pensar la naturaleza del dinero ha distanciado a las vertientes más importantes en la heterodoxia: algunos marxismos y las escuelas postkeynesianas.

Lawson entonces al pensar el dinero como objeto posicionado, pretende lograr la cuadratura del círculo: Poder integrar la naturaleza del dinero mercancía y la del dinero deuda, dos visiones que como hemos visto son contradictorias. El punto de partida es pensar que el dinero se integra en sistemas más complejos como objeto posicionado teniendo ciertas capacidades o funciones que ya posee antes de ser posicionado. La pregunta en clave ontológica que se hace Lawson es ¿Cuáles son las propiedades necesarias para que un objeto como el dinero antes de ser posicionado cumpla posteriormente sus funciones exitosamente? (Lawson, 2016).

Lawson propone priorizar la función de depósito de valor por encima de la de unidad de cuenta (dinero como deuda) y de la de medio de cambio (dinero como mercancía). Así, la condición fundamental para que el dinero pueda realizar con éxito sus funciones una vez posicionado, es que tenga a priori, una forma de valor que genere confianza.

Lawson resume su concepción de la naturaleza del dinero a través del posicionamiento social:

"El dinero se constituye donde es aceptado por una comunidad específica donde un objeto o cosa de valor se posiciona como una forma generalizada de valor, para funcionar como un medio general de pago, en condiciones de un sistema común o compartido de medición de valor igualmente aceptado y apropiadamente posicionado. El dinero simplemente es esa forma de valor posicionada" (Lawson, 2016, pág. 12)

A partir de esta estructura, busca mostrar como las teorías del dinero mercancía y del dinero crédito pueden articularse como casos particulares de esta meta concepción general. Así, por ejemplo, el crédito y la deuda pueden interpretarse como formas de valor que generan confianza y que se transfieren (cumpliendo la función de medio de pagos) y por lo tanto pueden ser posicionadas como dinero. En otras palabras, lo que se ha posicionado es un conjunto de relaciones sociales de débito y crédito. En el caso del dinero mercancía, sucede el mismo proceso de posicionamiento. Existe una mercancía que tiene una forma transferible de valor, y que es aceptada dentro de una comunidad como forma general de valor y que sirve como medio de pago (posicionamiento), en este caso desarrollando la función de medio de intercambio. Tenemos entonces que hay un valor prexistente con unas capacidades y propiedades que puede posicionarse en una estructura que subyace al mundo y que esta posición que existe independientemente de nosotros, le permite desplegar otras funciones.

Frente a la propuesta del posicionamiento social, Ingham (2017) crítica que no es posible que exista un valor que pre-exista al dinero. El dinero mismo es el surgimiento de este valor abstracto y como vimos, descansa en ultimo termino en elementos místicos o cuasireligiosos, como la fe generalizada. Por consiguiente, se rechaza el intento de 
interpretar el dinero como el dios Jano, teniendo dos cabezas que miran para dos horizontes opuestos. Desde la crítica de los teóricos del dinero crédito como Ingham, las dos naturalezas del dinero son irreconciliables, por consiguiente, ninguna construcción teórica puede hacerlas coexistir. Para él, el posicionamiento del valor en las dos teorías descansa sobre dos registros ontológicos diferentes. El problema es el uso de dispositivos analíticos como el posicionamiento social para explicar el dinero. En la próxima sección no se critica solo el uso sino la misma concepción ontológica.

\section{LA CRÍTICA A LA ONTOLOGÍA Y EL POSICIONAMIENTO SOCIAL}

Como señala Tsilipakos (2012), en los últimos años categorías como ontología, ontología social y status ontológico, han invadido el espectro de análisis en ciencias sociales y es importante preguntarse si esto ha mejorado la conversación respecto a las viejas maneras en que se debatían los problemas epistemológicos. ¿Ha consistido el razonamiento ontológico un avance o un retroceso?

Una de las críticas centrales a este tipo de proyectos ontológicos, es que proyectan sobre el mundo cierto tipo de estructuras que no tienen existencia más que como categorías discursivas. Cómo señala Rom Harré, curiosamente uno de los filósofos de la ciencia que influyó en Roy Bhaskar y su teoría realista de la ciencia de la que se deriva la ontología:

". . la realidad social se agota "por lo que hace la gente". Las reglas por las que lo gestionan no deben reincorporarse a un ámbito trascendente del cual ejercen su influencia benigna. ¿Qué realidad tienen? De nuevo debemos distinguir un modo de ser como inmanente en las prácticas, muchas de las cuales son discursivas, y un modo de ser como instrucciones concretas, que son reales como ejemplos de discurso. En resumen, la única realidad que tienen las normas y las reglas es del mismo estatus ontológico que las actividades que "gobiernan", es decir, los actos discursivos" (Harré, 2002, pág. 116)

En otras palabras, cuando hablamos del mundo social en términos ontológicos estamos otorgándole vida propia a estructuras que no existen sino como recursos discursivos. La identificación de estructuras independientes como las del posicionamiento social, no identifican las ultimas estructuras de la realidad, sino que proyectan esas estructuras como independientes en el mundo. Convierte entonces cuestiones epistemológicas y metodológicas en cuestiones ontológicas (falacia ontológica). Esto incluso vale para conceptos que usamos aceptando que tienen realidad independiente.

“... los únicos detalles poderosos que existen para que el mundo social exista son las personas. La noción de clase, por ejemplo, es un término taxonómico que no tiene poderes causales. No es una propiedad que simplemente se puede atribuir a un individuo en términos de sus capacidades ubicadas, sino que existe en la mente de los clasificadores y, por lo tanto, no es una propiedad del mundo social. Esta y otras macrocolectividades se consideran dispositivos retóricos con claras implicaciones para una teoría social, que tiene intenciones de transformación" (May \& Williams, 2002, pág.108)

Así, no explicamos que el caballo coma pasto porque es herbívoro. No se debe dar trascendencia a estructuras que solo usamos como maneras de hablar para algo que observamos. No debe pensarse que nuestras categorías taxonómicas son objetos que tienen existencia propia en el mundo sin ningún observador que las describa. Por 
supuesto, en la vida cotidiana no solemos pensar en el tipo de existencia que tienen nuestros conceptos y su relación con la realidad que experimentamos. El mundo que damos por sentado, es un mundo que ha sido depurado (Nuestras discusiones se dan sobre objetos o fenómenos sobre los que ya hay un camino trazado de sentido, pero discutirlo no implica negar a priori que ese sentido trazado no pueda ser pertinente). Por ejemplo, la noción de productividad no está en las cosas mismas, no tiene existencia ontológica, aunque en nuestra vida cotidiana lo parezca, es una categoría que señala un sentido y significado asociado a ciertas prácticas, pero podemos encontrar valioso discutir con este concepto y discutir como se mide y se constituye por los efectos prácticos y las disputas que hay en juego 5 .

En todo caso, no se debe perder de vista la observación de los biólogos chilenos Humberto Matuana y Francisco Varela "Todo lo dicho, es dicho por un observador que hace distinciones" (Maturana \& Varela, 1999, pág. 16). Esto por supuesto no significa que nuestras valoraciones o descripciones sean arbitrarias, pero en todo caso no hay una correspondencia univoca entre lo que decimos del mundo y lo que sería el mundo en sí mismo.

\section{EL DINERO BAJO LA ÓPTICA DEL ANTIESENCIALISMO CRÍTICO}

"Desde que la filosofía occidental, a partir de Grecia, se impuso como finalidad el conocimientoracional dela verdad y la tradición europea seapartódefinitivamente de la sabiduría como experiencia vital... es necesario dar consistencia a la antigua vía de la sabiduría, abriendo una posibilidad al pensamiento distinta a la que ha desarrollado la filosofía. En China, donde no se erigió el edificio de la ontología, el sabio, como se ha dicho de Confucio, no tiene ideas, ni prejuicios, puesto que parte del supuesto de que toda idea es ya algo que se superpone a la realidad. Por eso desconfía de las ideas, no quiere atarse a ellas, porque no sólo nos distancian de las cosas, sino que, además, al fijar y codificar el pensamiento, lo vuelven demasiado parcial, lo privan de su disponibilidad. No se trata por tanto de conocer definiendo los objetos, sino de tomar consciencia del fondo de la inmanencia que dispensa lo evidente: aquello que, precisamente por tenerlo siempre ante los ojos, no vemos, no alcanzamos a ver" (Jullien, 2008, pág. 130).

Como dice Schumpeter en su monumental obra, la historia del análisis económico,

"En la práctica, todos comenzamos nuestra propia investigación a partir del trabajo de nuestros predecesores, es decir, casi nunca comenzamos desde cero. Pero supongamos que comenzamos de cero, ¿cuáles son los pasos que debemos tomar? Obviamente, para ser capaces de plantearnos cualquier problema, primero debemos visualizar un conjunto distinto de fenómenos coherentes como

\footnotetext{
5. El filósofo greco-francés Cornelius Castoriadis, postuló que todos los trabajos deberían tener el mismo salario, idea que nuestro sentido común encontraría absurda. No obstante, si ponemos entre paréntesis lo aprendido y seguimos el consejo fenomenológico de "volver a las cosas mismas", lo que vemos en el mundo son una serie de personas que realizan una serie de procesos y producen un producto colectivamente (Esto a su vez también es una interpretación), por eso Castoriadis reivindica a Nietzsche y su "No hay hechos sino interpretaciones". El conocimiento es entonces circular, pero busca su autotrascendencia en una lógica parecida a la del dinero, como se explicó más arriba. Para ver los paralelos entre la lógica circular de distintos fenómenos, incluido el dinero, ver el libro Understanding origins de F. Varela y J.P. Dupuy (1988)
} 
un objeto valioso de nuestro esfuerzo analítico. En otras palabras, el esfuerzo analítico es necesariamente precedido por un acto cognitivo preanalítico que suministra la materia prima para el esfuerzo analítico. En este libro, este acto cognitivo preanalítico se llamará Visión". (Schumpeter, 1988, pág. 112)

"Comenzar de cero" es un recurso metodológico que debería ser más valorado y menos chocante para los académicos. Como bien lo dice Heidegger (2005), la tarea del pensar es la "devolverse con el pensar hacia lo ya pensado para estar al servicio de lo pensar" (Heidegger, 2005, pág. 43).Esta jugada cambia la manera de situarse en el mundo (o en un sentido más amplio de ser-en-el-mundo como gustaba decir a Heidegger), pues no aparece como dado. Esto cambia la manera de conversar y de hacer preguntas. Desde esta posible forma de auto interpretación en el mundo, la pregunta por la verdadera naturaleza del dinero es una pseudo-pregunta, pues estamos situados en un mundo que no está dado independientemente, sino que está coexistiendo, esta puesto en marcha.

Se acepta el desafío antiesencialista y su rechazo del ideal de encontrar la verdadera naturaleza del dinero. Sin embargo, en un espíritu pragmatista, se juzga las concepciones por las consecuencias que se producen. Se toma una analogía con la forma crítica que desarrolló Richard Rorty (1990) sobre la tesis de Paul Feyerabend (1978) en su libro La ciencia en una sociedad libre. Feyerabend sostiene que en una sociedad libre, la magia, la religión, entre otros sistemas de conocimiento, deberían tener la posibilidad de tener la misma voz que tiene la ciencia como voz más autorizada. Rorty concuerda en que el método científico a-priori no es en sí mismo superior a otro tipo de metodologías y que por lo tanto estas no se deberían restringir ex-ante, por razones de consistencia interna, externa, o cualquier otro criterio, pues podemos estar perdiéndonos de resultados o entendimientos valiosos, solo por que no se ajustan a requerimientos metodológicos. De hecho, por errores, accidentes e hipótesis ad-hoc, la ciencia también ha progresado (Feyerabend, 1978). No obstante, Rorty también señala que a posteriori los diferentes sistemas de conocimiento tienen diferentes alcances y la ciencia alcanza generalmente una conversación más sistemática, con mejores entendimientos y mejores resultados (Rorty, 1990). Una de las riquezas del pragmatismo epistemológico, es evitar a toda costa las verdades o naturalezas eternas y aceptar la multidiversidad de explicaciones a priori, pero a posteriori juzgar por las consecuencias que producen las concepciones.

Nos interesa pensar la naturaleza del dinero bajo estas coordenadas. Mas que afirmar como Lawson que el dinero per se no es crédito ni mercancía, es pertinente pensar el dinero como un espacio más abierto (su naturaleza es contingente) que cerrado (hay una verdadera naturaleza). No obstante, se crítica la concepción del dinero mercancía por el mundo que se ha diseñado a partir de su concepción, la manera en que se quiere llevar la realidad a la imagen de equilibrio que surge del trueque ${ }^{6}$.

Se utiliza el antiesencialismo frente al naturalismo en que nos han sumergido las argumentaciones tradicionales basadas en la imposibilidad de implementar otras políticas monetarias y fiscales, como si se tratara de leyes de la física. La manera de pensar la economía como un trueque y la forma en que se le traza un horizonte a la realidad a partir de esta imagen tiene consecuencias, pero que no son para nada leyes ineludibles.

\footnotetext{
6. Aquí no se discute la cuestión de si es posible construir otras posibilidades a partir de la concepción del dinero mercancía como lo postulan algunas vertientes heterodoxas, él foco está puesto sobre lo que se ha logrado construir con esta imagen del trueque.
} 
Se trata de desnaturalizar posiciones como la que restringe la capacidad del estado para intervenir (re)distributivamente y solo ve posible las intervenciones que aumentan la competencia, lo que tiene consecuencias entre otros para los trabajadores, los desempleados y los desposeídos. El llamado del antiesencialismo, es a cuestionar lo que auto trasciende como punto fijo. Sin embargo, el punto no es defender una posición antifundacionalista. Más bien, ver qué otras posibilidades se abren, lo que genera conflictos por el tipo de mundo o moneda en este caso que se quiere instituir. Así, los problemas epistemológicos son profundamente políticos. En este ensayo argumentamos a favor del dinero como relación social, no porque se pretenda señalar su verdadera esencia, sino por los mundos que permite desplegar teniendo como punto de valoración, la construcción de una sociedad que permita las condiciones de una vida vivible para todos los seres humanos, donde el horizonte sea un mundo común y no uno donde la competencia es el meta valor que guía las relaciones humanas. Esto es, una concepción de la política que cuestiona o aumenta la potencia de los dominados (los trabajadores, los desempleados, los desposeídos o "los que no tienen", según la expresión de Branco Milanovíc), en las relaciones de poder. Aunque estas categorías (dominantes y dominados) usualmente se han pensado en marcos esencialistas, acá se rescatan de manera contingente para señalar unos conflictos que no tienen "realidad ontológica", sino que constituyen maneras de referirnos a las cosas.

Comenzaremos entonces por señalar las consecuencias de la concepción de la moneda en el desarrollo de las zonas monetarias. Como lo ha señalado Charles Goodhart (1998), el enfoque de las zonas monetarias óptimas (ZMO) desarrollado por Robert Mundell, parte de la concepción de que el dinero es un numerario, que no distorsiona el mercado sino por el contrario su función principal es disminuir los costos de transacción. Desde esta perspectiva, una zona monetaria como la eurozona, a la que subyace una unión monetaria pero no política ni fiscal, no es problemática, pues el dinero no tiene relación profunda con la soberanía política y fiscal de un país. Así, una institución como el Banco Central Europeo (BCE) es independiente de cualquier gobierno. No obstante, ¿Qué pasa si las personas expresan sus deseos a través de instituciones democráticas, y estos no son los objetivos y operaciones del sistema europeo de bancos centrales (ECBS), ¿cómo de hecho sucedió en Grecia en el año 2015? Se tiene que se ve limitado el poder constituyente en nombre de supuestos intereses "supremos", como si fueran dados por los dioses o naturalizados. A su vez,

"los Estados miembros de la zona monetaria siguen teniendo responsabilidades fiscales; pero en el campo monetario, su estado ha cambiado a un nivel subsidiario, en el sentido de que, en una situación crítica, ya no pueden actuar con la autoridad monetaria para crear dinero, por ejemplo para financiar su deuda doméstica" (Goodhart, 1998, pág. 411).

Esto entonces genera una separación entre lo fiscal y la producción de dinero público. La concepción que se desprende del dinero mercancía, al no reconocer este vínculo entre soberanía política y creación de dinero, termina restando autonomía a los gobiernos soberanos. Algunos objetan que esta sujeción de la democracia y del poder de emisión es necesaria por qué algunos países "han vivido por encima de sus medios", pero esto no es el ser de las cosas, sino una lectura que es necesario desterrar señalando que está ligado a una concepción del dinero, en la que se perjudica a un gobierno soberano pues se reduce su poder y aumenta el de los financieros. La moneda como relación social al reconocer este vínculo permite mayor autonomía social. 
En segundo lugar, la concepción del dinero como relación social, permite visibilizar que las políticas monetarias no son neutrales ni en el corto ni en el largo plazo. Desde la perspectiva del dinero endógeno se puede señalar que la tasa de interés nominal es una variable distributiva (Rochon, 2017) y la inflación no es un fenómeno monetario, sino que es un conflicto entre grupos que poseen riqueza real o financiera y quienes no la poseen. Así, el dilema que enfrentan los bancos centrales es si optan por favorecer a alguno de estos grupos o se declaran neutrales en el conflicto distributivo entre rentas, salarios y beneficios. Las decisiones de tasa de interés a partir de la regla de Taylor, que por medio de las brechas de inflación y de producto y la tasa natural de interés juzgan la realidad según la norma de una economía en equilibrio como la del trueque, terminan tomando partido en el conflicto distributivo a expensas de los grupos no poseedores de riqueza real o financiera (Moreno, 2014). Podemos ver entonces como el rechazo de la neutralidad monetaria a largo plazo, postulado que se desprende del dinero mercancía, permite señalar que los regímenes de inflación objetivo y su procedimiento de elección de tasas de interés ocasionan la perdida de participación de los no poseedores de riqueza en el ingreso nacional.

En tercer lugar, desde la perspectiva del dinero como relación social puede señalarse que el desempleo es un fenómeno monetario, que está ligado a la demanda especulativa de dinero (Keynes, 1936). En otras palabras, el desempleo involuntario está ligado al atesoramiento de dinero como reserva de valor (Moreno, 2014). El dinero es fundamental para entender el desempleo y así rechazar conceptos como la tasa natural de desempleo, que rescatan la necesidad del desempleo involuntario y cuyo efecto práctico es disminuir el poder de los trabajadores (Kalecki, 1943). Todo esto en nombre de la inevitabilidad de no distorsionar el mecanismo equilibrador de mercado (horizonte trazado por el dinero mercancía).

Por último, puede señalarse que desde la concepción de la moneda endógena (relación social) los ahorros no son necesarios para la inversión. De hecho, son los ahorros los que necesitan ser financiados, pues en todo momento el dinero es una reclamación de alguien a otro alguien. No hay límites en la cantidad de financiamiento que se necesita para los grandes desafíos de la humanidad. Como dice Ann Pettifor:

"Esa es la buena noticia: un sistema monetario bien desarrollado puede financiar proyectos muy grandes, proyectos cuya financiación superaría con creces los ahorros totales de una economía, escondidos en alcancías u otras instituciones. Eso significa que una sociedad basada en un sistema monetario sólido podría 'permitirse' un sistema de educación y salud gratuito; podría financiar el apoyo para las artes y la defensa; podría enfrentar enfermedades o rescatar bancos en una crisis financiera. Si bien podemos estar escasos de los recursos físicos y humanos necesarios para transformar las economías de los combustibles fósiles, la sociedad nunca necesita estar corta de las relaciones financieras -los reclamos que hacemos entre nosotrosnecesarias para los cambios urgentes para garantizar que permanecemos en un medio ambiente habitable. Sin embargo, si un sistema monetario no se gestiona y opera en su lugar en beneficio de unos pocos, puede tener un impacto económico, político y ambiental catastrófico. (Pettifor, 2017, pág. 23)

Desde esta perspectiva se rechaza la escasez de dinero en la sociedad que lo que hace es aumentar el ejercicio de poder de los poseedores de riqueza real o financiera en contra de quienes no tienen.

Como se pudo ver en estos cuatros ejemplos, de una lista que puede aumentarse, el 
horizonte trazado por el dinero como relación social permite aumentar el espacio de poder de los que se encuentran en condiciones precarias.

\section{CONCLUSIONES}

Este artículo ha buscado rescatar y reformular una forma de pensar problemas epistemológicos en economía, específicamente en torno al debate de la naturaleza del dinero. En primer lugar, se presentaron las dos concepciones que han sido antagónicas sobre la naturaleza del dinero. La del dinero-mercancía y la del dinero-relación social. En esta parte se vio que uno de los rasgos fundamentales de las concepciones del dinero-mercancía es la imagen del trueque, lo que significa que el dinero se introduce posteriormente al orden mercantil, lo que va tender a resaltar la estabilidad del mecanismo de mercado y la neutralidad del dinero a largo plazo, pues el dinero al ser un tipo de bien no debería distorsionar los intercambios mercantiles. Por el contrario, las concepciones del dinero como relación social señalan que el dinero no es una cosa sino relaciones sociales entre agentes, y que el dinero es el que posibilita el orden mercantil. Se tiende además a subrayar la importancia del vínculo entre Estados soberanos y dinero.

Posteriormente se revisó una de las recientes propuestas ontológicas que trata de articular las dos naturalezas del dinero. Se trata de hacerlas coexistir por medio de la figura del posicionamiento social, una herramienta que identifica estructuras que existen en el mundo y que permiten generar lo real. Esta idea nos dice que el dinero no es en sí mismo mercancía o crédito, sino que estas formas son posicionamientos sociales del dinero.

Por último, dentro del espíritu de corrientes como la fenomenología y el pragmatismo, se despliega lo que aquí llamamos un antiesencialismo crítico. Al desestabilizar la separación entre cuestiones de hecho y de valor, no se pregunta por la verdadera naturaleza del dinero, sino que nos ocupamos de las consecuencias que se producen o se pueden producir bajo cada concepción teniendo como criterio la perspectiva de los que tienen menor posibilidad de ejercer poder en las diferentes relaciones sociales. Por ejemplo, a nivel de las zonas monetarias óptimas, la concepción del dinero-relación social al señalar un vínculo entre dinero y soberanía política aumenta el margen de autonomía para los ciudadanos. Lo mismo puede decirse del rechazo a la neutralidad del dinero, que puede disminuir el grado de autonomía de los no poseedores de riqueza. A su vez, los trabajadores y desempleados se pueden ver empoderados con políticas monetarias que rechazan la neutralidad del dinero. El punto central es que el debate sobre la naturaleza del dinero importa, no porque corresponda a un debate sobre la idea verdadera sobre la naturaleza del dinero, sino porque estas interpretaciones disponen mundos, señalan conflictos y generan consecuencias. Aunque con una intención diferente a la de Marx, podemos retomar sus palabras de las Tesis sobre Feuerbach "Es en la práctica donde el hombre tiene que demostrar la verdad” (Marx, 1990, pág. 5).

\section{REFERENCIAS BIBLIOGRÁFICAS}

Aglietta, M., \& Orleán, A. (1988). La violencia de la moneda. Mexico: Siglo XXI.

Aglietta, M., Ponsot, J.-F., \& Ould Ahmed, P. (2016). Monnaie entre dettes et souveraineté. Paris: Éditions Odile Jacob.

Baskhar, R. (1975). A realist theory of science. New York: Routledge. 
Colander, D. (2000). The death of neoclassical economics. Journal of the History of Economic Thought Volume 22, Issue 2, 127-143.

Davidson, P. (1972). Money and the real world. London: Palgrave.

Davidson, P. (2007). Money And Keynes. En P. Arestis, \& M. Sawyer, A hanbook of alternative monetary economics (págs. 139-152). Chentelham: Edward Elgar.

Dequech, D. (2013). Is money a convention and/or a creature of the state? the convention of the acceptability, the state, contracts and taxes. Journal of Post Keynesians Economics, Vol 36 No2. 251251-275.

Dodd, N. (2014). The social life of money. New Jersey: Princeton Press.

Dupuy, J.-P. (2012). L'Avenir de l'économie: Sortir de l'écomystification. Paris: Flammarion.

Escobar-Espinoza, A., \& Angulo-Pico, G. (2015). Panorama Economico in the context of the international visibility of scientific journals. Panorama Económico, 23, 11-13. DOI: https://doi.org/ 10.32997/2463-0470-vol.23-num.1-2015-1733

Escobar-Espinoza, A., Guevara-Castañeda, D., \& Uribe-Veloza, M. (2017). Modelos Computacionales y Análisis de la Política Económica en Colombia. Panorama Económico, 25(4), 535-558. DOI: https://doi.org/10.32997/2463-0470-vol.25-num.4-2017-2088

Felipe, J. (2009). Inclusive Growth, Full Employment, and Structural Change: Implications and Policies for Developing Asia. Cambridge: Anthem Press.

Feyerabend, P. (1978). La ciencia en una sociedad libre. México: Siglo XXI.

Fontana, G. (2004). Hicks on monetary theory and history: Money as endogenous money. Cambridge journal of economics, 28, 73-88.

Girard, R. (1982). La violencia y lo sagrado. Barcelona: Anagrama.

Goodhart, C. (1998). The two concepts of money: implications for the analysis of optimal currency areas. european journal of political economy. Vol 14, 407-432.

Graeber, D. (2011). Debt: The First 5,000 Years. New York: Melville House Publishing.

Hahn, F. (1965). On some problems of proving the existence of an equilibrium in a monetary economy. En F. Hahn , \& F. Brechling, The Theory Of interest Rates (págs. 126-135).Macmillan.

Heidegger, M. (2005). Que significa pensar? Madrid: Trotta.

Ingham, G. (2004). Nature Of Money. London: Routledge.

Ingham, G. (2017). A critique of Lawson's 'Social positioning and the nature of money. Cambridge Journal of Economics 2017, 1-14.

Jevons, S. (1875). Money and the mechanism of exchange. New York: Cosimo Classics.

Jullien, F. (2008). Un sabio no tiene ideas. Madrid: Siruela.

Kalecki, M. (1943). Aspectos políticos del pleno empleo. Political Quarterly, vol 14, 347-356.

Keen, S. (2011). Debunking economics. Madrid: Cápitan Swing.

Lapavitsas, C. (2003). Social Foundations of Markets, Money and Credit. London: Routledge.

Lavoie, M. (2014). Post-keynesian economics. London: Edward Elgar. 
Lawson, T. (1997). Economics and reality. Abingdon: Routledge.

Lawson, T. (2016). Social positioning and the nature of money. Cambridge Journal of economics, 40, 961-996.

Marchart, O. (2009). El pensamiento político posfundacionalista . Bogotá: Fondo de cultura económica.

Martin, F. (2013). Money. The Unauthorised Biography. London: Vintage Books.

Marx, K. (1990). Tesis Sobre Feuerbach. Madrid: Grijalbo.

Maturana, H., \& Varela, F. (1999). El arbol del conocimiento. Santiago de chile: Lumen.

Menger, C. (1892). On the Origin of Money. The Economic Journal, 239-255.

Mirowski, P., \& Nik-Khah, E. (2017). The knowledge we have lost with information. New York: Oxford University Press.

Moreno, A. (2014). Política monetaria y distribución funcional del ingreso. Documento escuela de economía No. 50, 1-39.

Orléan, A. (2011). L’Empire de la valeur. Refonder l'économie. París: Seuil.

Parguez, A., \& Seccareccia, M. (1999). Credit theory: monetary circuit approach . En J. Smithin, What is money? (págs. 101-123). New York: Routledge.

Pettifor, A. (2017). The production of money: How to break the power of banks. New York: Verso. Rochon, L.-P. (2017). Rethinking monetary policy. En L.-P. Rochon, \& S. Rossi, A modern guide for rethinking economics (págs. 120-130). London: Edward Elgar.

Sala-i-Martin, X. (2015). Economía en colores. Barcelona: Conecta.

Schumpeter, J. (1988). Historia del analisis económico. Madrid: Ariel.

Searle, J. (2010). Creando el mundo social. La estructura de la civilización humana. Madrid: Paidos.

Simmel, G. (2014). Filosofía del dinero. Madrid: Capitán swing.

Smithin, J. (2013). Requirements of a Philosophy of Money And Finance. En J. Pixley et al., Financial Crises and the Nature of Capitalist Money (págs. 19-31). London: Palgrave Macmillan.

Tsilipakos, L. (2012). The Poverty of Ontological Reasoning. Journal for the Theory of Social Behaviour , 1-19.

Vergnhanini, R., \& De Conti, B. (2017). Modern Monetary Theory: a criticism from the periphery. Brazilian keynesian review.

Vergara-De la Ossa, R., Londoño-Vega, C., Pérez-Benítez, N., \& Torres-Castellar, R. (2015). La adopción de las Normas Internacionales de Información Financiera en Colombia. Panorama Económico, 23, 119-132. DOI: https://doi.org/10.32997/2463-0470-vol.23-num.1-2015-1382

Weber, M. (2006). Economía y sociedad. México: Fondo de cultura económica.

Williamson, O. (1975). Markets and Hierarchies: Analysis and Antitrust. New York: Free Press.

Zelizer, V. (2011). El significado social del dinero. Buenos Aires: FCE.

Zelmanovitz, L. (2016). The ontology and function of money. The philosophical fundamentals of monetary institutions. Lanham: Lexington books. 